\title{
Convergência Tecnológica Aplicada a Documentos de Patentes sobre Técnicas de Produção de Mudas de Cana-de-Açúcar Através da Análise de Coclassificação
}

\author{
Technological Convergence Applied to Patent Documents on Sugarcane \\ Seedlings's Production Techniques through Co-classification Analysis
}

\author{
Cecilia Hasner ${ }^{1}$ \\ Eduardo Winter ${ }^{2}$ \\ Araken Alves de Lima ${ }^{2}$ \\ ${ }^{1}$ Prospective Inovação Tecnológica e Ambiental Ltda-ME \\ ${ }^{2}$ Instituto Nacional da Propriedade Industrial - INPI
}

\begin{abstract}
Resumo
Estudos de convergência tecnológica geralmente utilizam análises complexos, tais como cocitações, co-words, coautoria, envolvendo artigos e documentos de patentes. Este trabalho descreve uma metodologia simples a ser aplicada a estudos de convergência tecnológica pela análise de coclassificação de documentos de patentes. Foi realizado um mapeamento tecnológico sobre a produção de mudas de cana-de-açúcar através de uma busca avançada na base Derwent Innovation Index no período de 2000 a 2016. Foram calculadas as taxas de convergência entre campos tecnológicos de uma mesma seção (intrasseção) e entre seções diferentes (interseção) das classificações Derwent. Os resultados apontam para taxas de convergência relativamente baixas, chegando a 8,36\% para convergências intrasseção em 2014 e 5,81\% para convergências interseção em 2012. As classificações do Derwent predominantes foram na indústria da fermentação, biotecnologia e cultivo vegetal, associadas às técnicas de produção de mudas por engenharia genética/melhoramento vegetal e controle de doenças e pragas. As tecnologias convergentes encontramse em áreas de ponta, tais como a biotecnologia, que precisam da complementaridade disciplinar no processo de inovação para alcançar ganhos de produtividade agrícola, redução de custos e inovações no melhoramento vegetal. A contribuição deste trabalho está na descrição de uma metodologia que pode ser aplicada a outros tipos de classificação de patentes em diferentes áreas tecnológicas.
\end{abstract}

Palavras-chave: Convergência tecnológica. Patentes. Coclassificação. Mudas de cana-de-açúcar.

\begin{abstract}
Technological convergence studies generally use complex analyzes, such as co-citations, co-words, co-authorship, involving papers and patent. This paper describes a simple methodology to be applied to studies of technological convergence by the co-classification analysis of patent documents. A technological mapping was carried out through an advanced search in the Derwent Innovation Index base about the production of sugarcane seedlings between 2000 and 2016. The technological convergence rates between technological fields of the same section (intra-section) and between different sections (inter-section) of the Derwent classifications were calculated. The results point to relatively low convergence rates, reaching $8.36 \%$ for intra-section convergences in 2014 and $5.81 \%$ for inter-section convergences in 2012. The predominant Derwent classifications were the fermentation, biotechnology and plant crop industry, associated to the techniques of production of seedlings by genetic engineering / plant breeding and control of diseases and pests. Converging technologies are in cutting-edge areas such as biotechnology, which need disciplinary complementarity in the innovation process to achieve agricultural productivity gains, cost savings, and innovations in plant breeding. The contribution of this work is in the description of a methodology that can be applied to other types of patent classification in different technological areas.
\end{abstract}

Keywords: Technological convergence. Patents. Co-classification. Seedlings of sugarcane. 


\section{Introdução}

Existe uma carência de estudos voltados a compreender melhor o elo da cadeia produtiva que engloba a produção das mudas e melhoramento da cana-de-açúcar no setor canavieiro e que mostrem a complexidade de tecnologias envolvidas no processo de produção de mudas. No Brasil, a produção de mudas de cana-de-açúcar tem variado ao longo do tempo, sendo a forma tradicional realizada a partir do corte da cana em seções, posteriormente depositadas em sulcos. Por outro lado, com a introdução da mecanização na lavoura, houve necessidade de melhorar a produtividade de cana por hectare, onde a produção de mudas com técnicas de cultivo de tecidos e encapsulamento do material embrionário tem levado à produção de "sementes artificiais"1. Estas mudanças tiveram início na última década e ainda não se consolidaram no mercado nacional, ressaltando a dificuldade tanto no desenvolvimento tecnológico de mudas de cana quanto na inserção deste produto, pelos custos de produção, armazenamento, logística, entre outros.

Entende-se como convergência a sinergia entre segmentos diferentes, como uma sobreposição de conhecimentos, trajetórias tecnológicas, segmentos industriais, mercado ou de serviços para produzir um novo produto (SONG; ELVERS; LEKER, 2017). Alguns autores fazem a diferenciação entre convergência e fusão, pela qual a convergência se refere a um processo no qual os "objetos" se movem de seus pontos iniciais para um lugar novo e comum, enquanto a fusão descreve um processo onde os objetos de diferentes pontos se fundem no mesmo lugar de pelo menos um dos objetos (CURRAN; BRÖRING; LEKER, 2010). Segundo Curran, Bröring e Leker (2010), é possível diferenciar quatro tipos de convergência: i) convergência científica, onde diferentes disciplinas começaram a citar-se entre uns e outros e a colaborar; ii) convergência tecnológica, onde a distância entre a ciência aplicada e o desenvolvimento tecnológico diminui; iii) convergência de mercado, a qual implica na combinação de novos produtos no mercado; e iv) convergência industrial, onde ocorre a fusão de segmentos industriais ou empresariais diferentes.

Uma revisão sistematizada das metodologias empregadas nos estudos de convergência tecnológica foi realizada pelos autores Choi, Jeong e Kim (2015), concluindo que a metodologia pela análise de coclassificação de documentos de patentes é a mais efetiva pela característica de que cada documento de patente possui uma classificação específica para seu domínio tecnológico (CHOI; JEONG; KIM, 2015; JEONG; KIM; CHOI, 2015). Estes autores descreveram as taxas de convergências tecnológicas utilizando a classificação internacional de patentes (IPC em inglês) em mais de um milhão de patentes depositados no Escritório de Patentes da Coreia no período de 1996 a 2010 (JEONG; KIM; CHOI, 2015), e notaram que a taxa de convergência tecnológica começou a aumentar visivelmente no início dos anos 2000 , independentemente do nível de identificação da convergência tecnológica, o que coincide com as políticas nacionais de fomento à ciência e tecnologia. As taxas de convergência tecnológica são inferiores a 3\% antes de 2002, mas atingem 19,2\% para convergências entre campos tecnológicos da mesma seção, e 33,0\% para convergências entre campos tecnológicos de seções diferentes em 2010. Os autores concluem dizendo que "a universalização da convergência tecnológica pode continuar

${ }^{1}$ Define-se como semente artificial o encapsulamento de qualquer tecido embrionário em estágio avançado de desenvolvimento, com capacidade de ser armazenado e de desenvolver uma planta completa in vitro e em campo (in vivo) (SNYMAN et al., 2011). 
pelo menos no futuro próximo, a menos que o atual sistema de inovação tecnológica enfrente mudanças drásticas de um novo tipo" (JEONG; KIM; CHOI, 2015, p. 853).

A análise de convergência tecnológica por coclassificação dos documentos de patente parte do pressuposto que uma patente com múltiplas indexações de campos tecnológicos diferentes representa uma convergência tecnológica, uma vez que um documento de patente possui conhecimento tecnológico baseado em domínios de tecnologia heterogêneos (JEONG; KIM; CHOI, 2015).

O presente estudo tem como objetivo principal realizar uma pesquisa para a convergência tecnológica utilizando como metodologia a coclassificação de patentes, tendo como principal objetivo analisar se existe complementaridade de domínios tecnológicos vinculados com as técnicas de produção de mudas de cana-de-açúcar ao longo do tempo, especificamente para o período de 2000 a 2016. O artigo está dividido em três partes: a primeira aborda a metodologia adotada para o levantamento dos documentos de patentes e seu tratamento, e depois a descrição da metodologia para calcular as convergências tecnológicas; a segunda parte são os resultados, divididos em seções. A primeira seção mostra o número total de documentos de patentes e o volume de classificações Derwent indexadas em cada documento. A segunda seção mostra a relação entre a classificação Derwent de patentes e a descrição das técnicas de produção de mudas de cana-de-açúcar através da rede de relacionamento. A terceira seção mostra os resultados das taxas de convergência intrasseção e interseção para o período de 2000 a 2016. A terceira parte é destinada às conclusões e encaminhamentos do trabalho.

\section{Metodologia}

A metodologia adotada para medir a convergência tecnológica se baseou em trabalhos recentes dos coreanos Choi, Jeong e Kim (2015) e Jeong, Kim e Choi (2015), que descrevem o uso das indexações de patentes por domínios tecnológicos de forma precisa e confiável. A metodologia pode ser dividida em quatro etapas:

\subsection{Levantamento dos Documentos de Patentes sobre a Produção de Mudas de Cana-de-Açúcar}

Esta etapa foi realizada em janeiro de 2017, utilizando-se a base do Derwent Innovation Index, da Thomsom Reuteurs, disponível na base de dados tecnológicos do Periódicos Capes, utilizando-se as palavras chaves sugarcane, seed, vegetative propagation, Sacharum, junto com a classificação internacional A01H (Novas Plantas ou Processos para Obtenção das Mesmas; Reprodução de Plantas por Meio de Técnicas de Cultura de Tecidos) no período de 2000 a 2016. Foram utilizados operadores booleanos e truncadores.

\subsection{Tratamento dos Dados}

Os dados foram exportados para uma planilha Excel, separados conforme o ano de publicação e identificadas todas as classificações Derwent indexadas por documento de patente. Estas foram ordenadas primeiramente pelo número de indexações e depois pelo tipo de convergência tecnológica: intra e interseção, explicado mais adiante. 
Ademais, os documentos foram agrupados segundo as técnicas de produção de mudas de cana-de-açúcar, descritas a seguir:

a) Recipiente de Mudas - contêiner de mudas de plantas;

b)Semente Artificial - relacionada com cana-de-açúcar;

c) Criopreservação - técnica de preservação do material propagativo vegetal;

d)Embriogenese/Micropropagação - técnicas de cultivo de tecido para produção de mudas;

e) Pesquisa/Invenção sobre cadeia produtiva de cana-de-açúcar - técnicas não relacionadas diretamente com a produção de mudas, mas que envolvem elos da cadeia produtiva do setor canavieiro;

f) Equipamento ou Dispositivo - inclui máquinas plantadeiras utilizadas na lavoura da cana;

g) Métodos/Pesquisa Propagação vegetativa - métodos de produção de mudas por propagação vegetativa não envolvendo cultivo de tecidos;

h)Pesquisa/Controle sobre doenças e pragas - utilização de biocidas, estimulantes de crescimento utilizados na produção e conservação das mudas;

i) Engenharia Genética ou Melhoramento - descrição de técnicas de engenharia genética ou de melhoramento vegetal;

j) Outros - documentos que não estavam relacionados com a propagação vegetativa, como por exemplo, a produção de etanol a partir da cana-de-açúcar.

\subsection{Análise das Redes de Relacionamento}

Para compreender a relação entre a descrição das técnicas de produção de mudas de cana-de-açúcar e as classificações de patentes, utilizaram-se programas de computador livre, tais como o NodeXL (SMITH et al., 2010) e o VOSviewer (VAN ECK; WALTMAN, 2017), este último recomendado para a visualização de indicadores relacionais (network) dos repositórios do Web of Science e do Derwent Innovation Index (RUAS; PEREIRA, 2014).

\subsection{Análise das Taxas de Convergência Tecnológica}

A análise das taxas de convergência tecnológica sobre técnicas de produção de mudas de cana-de-açúcar foi realizada utilizando-se a Classificação do Derwent, devido a grande vantagem de todos os documentos serem classificados pela base com os mesmos critérios, evitando assim possíveis divergências entre os escritórios de patentes na indexação dos documentos (JANNUZZI; AMORIM; SOUZA, 2007). A classificação Derwent possui somente três seções: Química (A-M), Engenharia (P-Q) e Eletroeletrônico (S-X).

A convergência intrasseção refere-se a documentos de patente indexados em diferentes campos tecnológicos da mesma seção, enquanto que a convergência interseção se refere a documentos de patente indexados em diferentes seções da Classificação Derwent. A definição conceitual está representada na Figura 1, a qual mostra alguns exemplos das Classificações Derwent do lado esquerdo nas três seções, seguidos de cinco exemplos de indexações de do- 
cumentos de patentes em códigos Derwent e quais documentos possuem (SIM) ou não (NÃO) convergências intrasseção e interseção.

O grau de convergência, tanto intra como inter, é calculado a partir da equação 01:

Grau de Convergência Tecnológica $=\frac{\text { Número de Publicações Técnicas convergentes }}{\text { Número Total de Publicações Técnicas }}$

Figura 1 - Esquema representativo sobre a definição de convergência tecnológica através da análise de coclassificação utilizando a Classificação Derwent

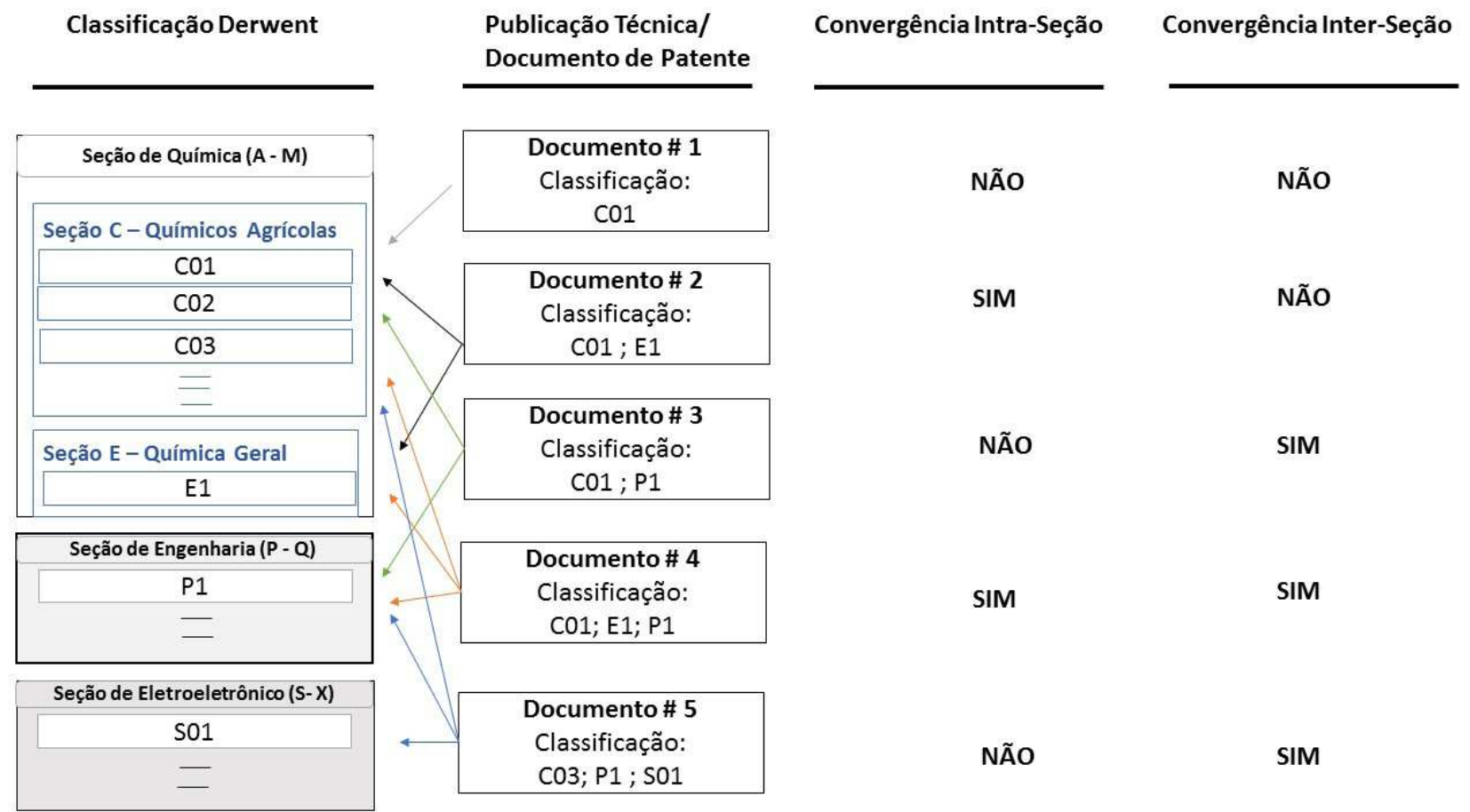

Fonte: Adaptado pelos autores deste artigo dos trabalhos de Choi, Jeong e Kim (2015) e Jeong, Kim e Choi (2015)

\section{Resultados e Discussão}

\subsection{Número Total de Documentos de Patentes e Frequência das Classificações Derwent}

A Tabela 1 mostra os resultados referentes ao total de classificações de patentes indexadas pelo Derwent Innovation Index, separados por seções. Observa-se que 73,4\% dos documentos de patentes possuem indexações na seção de Química, com uma maior frequência das classificações D16 e C06, referentes à indústria da fermentação e Biotecnologia, genética vegetal, vacinas veterinárias, respectivamente. A seção de Engenharia foi majoritariamente da classificação P13, Cultura vegetal, produtos lácteos, a qual tem coerência com o objeto de estudo, que é a produção de mudas de cana-de-açúcar. A seção de Eletroeletronicos foi pouco expressiva, somente com $2,3 \%$ dos documentos de patentes indexados. 
Tabela 1 - Descrição das principais classificações de patentes do Derwent indexadas nos documentos de patentes sobre técnicas de produção de mudas de cana-de-açúcar representadas na Figura 3

\begin{tabular}{|c|c|c|}
\hline Classificação & DESCRIÇÃo & FrEQUÊNCIA Total \\
\hline A-M & Seção de Química & \\
\hline D16 & Indústria da Fermentação & 1.491 \\
\hline $\mathrm{C} 06$ & Biotecnologia, genética vegetal, vacinas veterinárias & 1.362 \\
\hline $\mathrm{C} 02$ & Heterocíclicos & 330 \\
\hline $\mathrm{C} 03$ & $\begin{array}{l}\text { Outros compostos orgânicos ou inorgânicos } \\
\text { e misturas multicomponentes }\end{array}$ & 253 \\
\hline A97 & Produtos diversos não especificados em outro lugar & 135 \\
\hline D13 & Outros gêneros alimentícios e tratamento & 118 \\
\hline B04 & $\begin{array}{c}\text { Produtos naturais e polímeros, testes, compostos } \\
\text { de estrutura desconhecida }\end{array}$ & 102 \\
\hline $\mathrm{C} 05$ & Controle Biológico & 99 \\
\hline $\mathrm{C04}$ & Fertilizantes incluindo ureia e a produção de $\mathrm{H} 3 \mathrm{PO} 4$ & 69 \\
\hline $\mathrm{C} 07$ & Equipamentos, fórmulas & 44 \\
\hline \multirow[t]{3}{*}{$\mathrm{C} 01$} & Organophosphorus, organometallic & 43 \\
\hline & Demais classificações da seção de Química & 453 \\
\hline & Total da seção de Química & $4.499(73,6 \%)$ \\
\hline $\mathrm{P}-\mathrm{Q}$ & Seção de Engenharia & \\
\hline $\mathrm{P} 13$ & Cultura vegetal, produtos lácteos & 1.249 \\
\hline \multirow[t]{3}{*}{$\mathrm{P} 11$} & Plantio, trabalho de solo & 163 \\
\hline & Demais classificações da seção de Engenharia & 65 \\
\hline & Total da seção de Engenharia & $1.477(24,2 \%)$ \\
\hline S-X & Seção Eletroeletrônico & \\
\hline T01 & Computadores digitais & 51 \\
\hline \multirow[t]{4}{*}{ T04 } & Equipamento Periférico de Computador & 36 \\
\hline & Demais classificações da seção de Eletroeletrônico & 52 \\
\hline & Total da seção de Eletroeletrônico & $139(2,3 \%)$ \\
\hline & Total das Indexações & $6.115(100 \%)$ \\
\hline
\end{tabular}

Fonte: Tradução própria da base Derwent Innovation Index

A Figura 2 mostra um resumo do número total de indexações das classificações dos documentos de patente recuperados no período de 2000 a 2016. Foram 1.999 documentos de patente, abrangendo 6.115 classificações Derwent, dos quais $12 \%$, ou 240 documentos de patentes, tiveram somente uma indexação e sua grande maioria, $88 \%$, correspondendo a 1.759 documentos, foi indexada com mais de uma Classificação Derwent. A partir deste universo foi possível analisar as taxas de convergência tecnológica intra e interseção sobre as técnicas de produção de mudas de cana-de-açúcar, onde o valor zero (0) corresponde a nenhuma convergência e dois (2) seria o valor mínimo de convergência entre dois campos tecnológicos 
distintos. O gráfico do lado esquerdo mostra a porcentagem das indexações dentro da mesma seção (intrasseção), onde é possível notar que $62 \%$ foram compostas somente por duas indexações, $13 \%$ com três indexações e $5 \%$ com quatro indexações (Figura 2). Do lado direito está representada a frequência das indexações realizadas entre seções diferentes (interseção), sendo que $53,5 \%$ dos documentos foram indexados em duas seções e somente 4,5\% tiveram três seções indexadas, o que já mostra um volume pequeno de interações multidisciplinares para a produção de mudas de cana-de-açúcar.

Figura 2 - Porcentagem dos documentos de patente sobre técnicas de produção de mudas de cana-de-açúcar com uma ou mais indexações da Classificação Derwent e a porcentagem do número de campos tecnológicos indexados intrasseção e interseção. N=1999. Período: 2000-2016

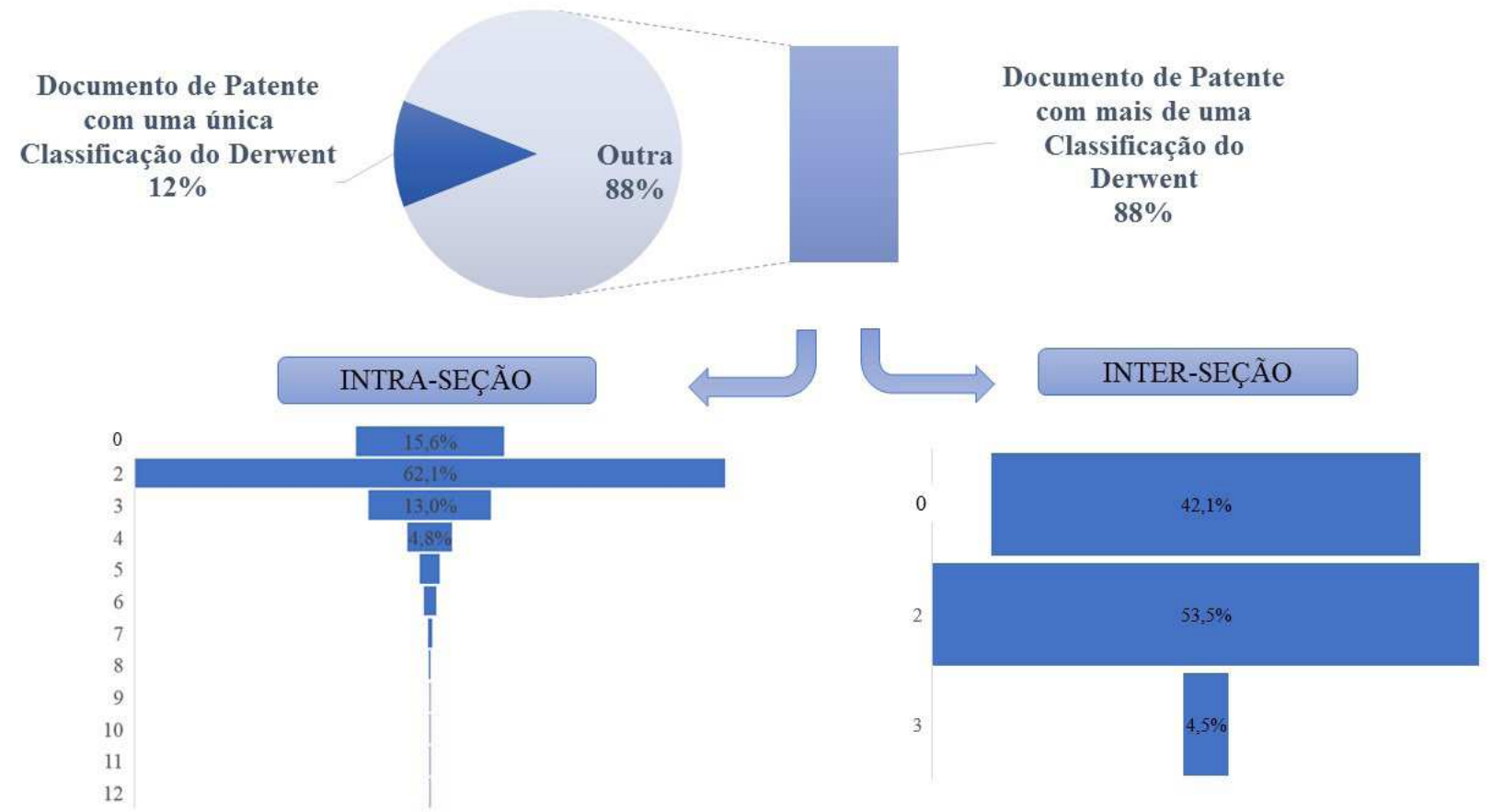

Fonte: Elaborada pelos autores deste artigo com base no Derwent Innovation Index

\subsection{Relação Entre Classificação de Patentes e Descrição das Técnicas de Produção de Mudas de Cana-de-Açúcar}

Foi realizada uma análise da relação entre as 6.115 classificações de patentes indexadas pelo Derwent e a descrição das técnicas de produção mencionadas na metodologia. $\mathrm{O}$ resultado está representado na figura 3, através de uma rede de relacionamento, onde a distância geodésica média foi de 2,49 e o grau de centralidade média (proximidade) de 6,847 (Tabela 2), mostrando ser uma rede bastante esparsa. Destaca-se a relação entre os documentos de patentes sobre técnicas de engenharia genética ou melhoramento vegetal e as Classificações do Derwent referentes às seções de Química e Engenharia, destacando-se os domínios de biotecnologia, da indústria da fermentação e o cultivo vegetal (C06+D16+P13) (Tabela 1). A criopreservação se relaciona diretamente com a classificação $\mathrm{C} 06$, para processos biotecnológicos. A seção de Eletroeletrônicos, representada pela classificação T01 (Tabela 1), aparece vinculada com a engenharia genética e melhoramento, e contempla basicamente a computação digital, muito 
utilizada nas modelagens e simulação de processos biotecnológicos. As técnicas de controle de doenças e pragas aparecem mais relacionadas com as classificações da seção de Química, tais como a C02, C03 e C05 (Figura 3, Tabela 1). Estes resultados mostram coerência entre a classificação indexada e as técnicas relacionadas com a produção de mudas de cana-de-açúcar.

Figura 3 - Rede de relacionamento entre as classificações de patentes indexadas pelo Derwent e a descrição das técnicas de produção de mudas de cana-de-açúcar. $\mathrm{N}=6.115$. Período: 2000-2016

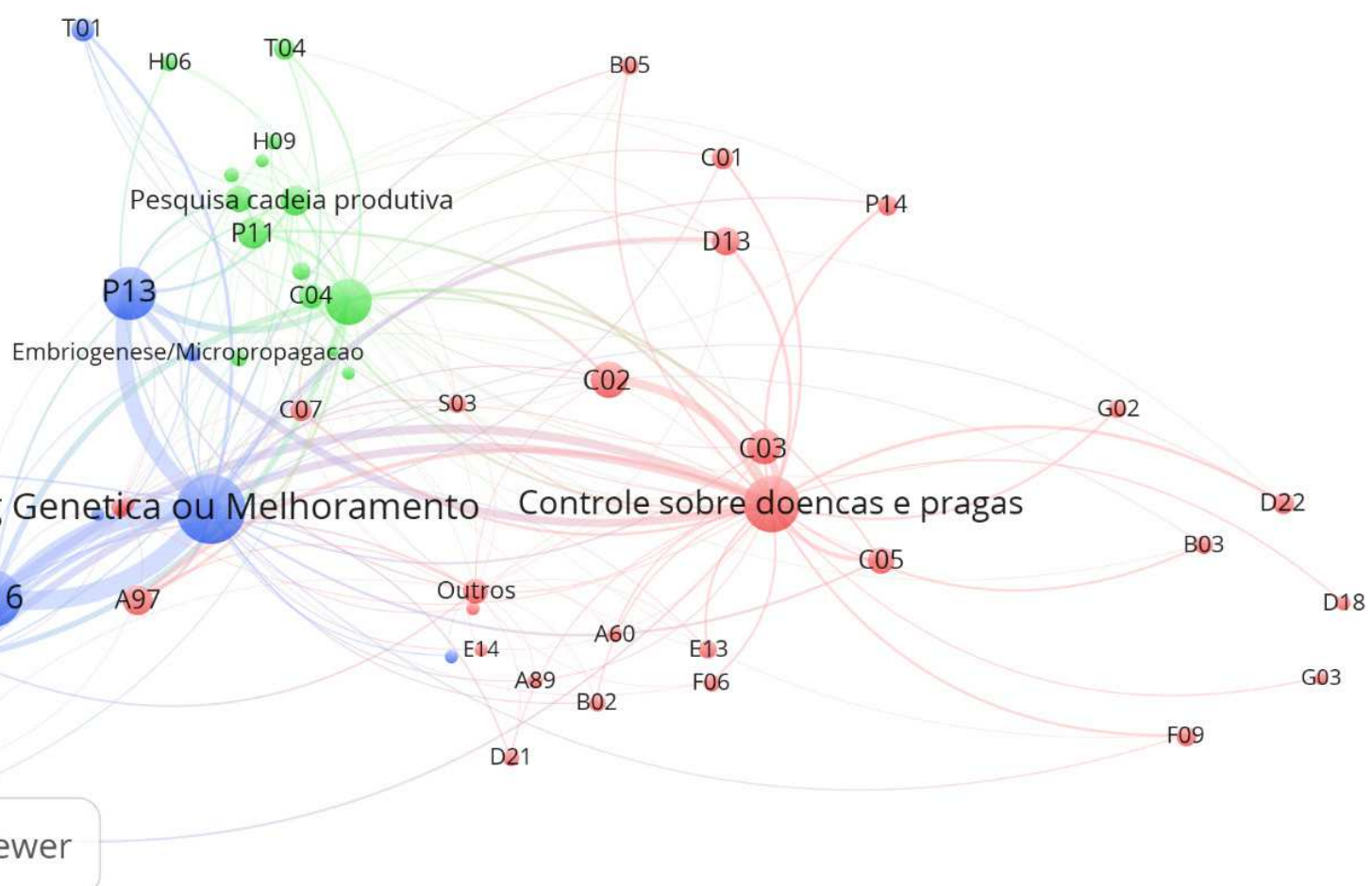

Fonte: Elaborada pelos autores deste artigo com base no Derwent Innovation Index. Programas de computador: NodeXL e VOSviewer

Tabela 2 - Indicadores de redes de relacionamento

\begin{tabular}{cc} 
Métricas do Gráfico de Relacionamento & ValoR \\
Tipo de Gráfico & Sem direção \\
Número de Vértices & 98 \\
Número Total de Edges sem duplicatas & 243 \\
Distância Geodésica Máxima (Diâmetro) & 5 \\
Distância Geodésica Média & 2,499792 \\
Densidade da rede & 0,051125605 \\
Grau de centralidade media (proximidade) & 6,847 \\
\hline
\end{tabular}

Fonte: NodeXL versão 1.0.1.399

A Tabela 3 mostra a frequência do número de classificações indexadas pelo Derwent segundo as técnicas de produção de mudas de cana-de-açúcar, mostrando que o maior volume de 
documentos de patentes encontra-se nas técnicas de engenharia genética e melhoramento e de controle de doenças e pragas. Ademais, o número de classificações indexadas por documento concentrou-se em duas e três classificações Derwent, o que permite a análise das convergências tecnológicas, descrita na próxima seção.

Tabela 3 - Número de classificações de patentes indexadas pelo Derwent por documento de patente segundo a descrição das técnicas de produção de mudas de cana-de-açúcar. N=1999. Período: 2000 2016

\begin{tabular}{|c|c|c|c|c|c|c|c|c|c|c|c|c|}
\hline \multirow{2}{*}{ Descrição da TeCnologia } & \multicolumn{11}{|c|}{ Número de Classificações por Documento de Patente } & \multirow{2}{*}{ TOTAL } \\
\hline & $\mathbf{1}$ & 2 & 3 & 4 & 5 & 6 & 7 & 8 & 9 & 10 & 12 & \\
\hline Recipiente de Mudas & & & 1 & & & & & & & & & 1 \\
\hline Semente Artificial & & & 1 & & & & & & & & & 1 \\
\hline Criopreservação & & 1 & 2 & & & & & & & & & 3 \\
\hline $\begin{array}{l}\text { Embriogenese/ } \\
\text { Micropropagação }\end{array}$ & & 1 & 3 & 1 & & & & & & & & 5 \\
\hline Outros & 1 & 3 & 12 & 3 & 1 & & & & & & & 20 \\
\hline $\begin{array}{l}\text { Pesquisa/Invenção sobre cadeia } \\
\text { produtiva de cana-de-açúcar }\end{array}$ & 4 & 14 & 27 & 13 & & & 2 & & & & & 60 \\
\hline Equipamento ou Dispositivo & 7 & 14 & 25 & 9 & 3 & 2 & 1 & 1 & & & & 62 \\
\hline $\begin{array}{l}\text { Métodos/Pesquisa } \\
\text { Propagação vegetativa }\end{array}$ & 27 & 58 & 86 & 28 & 14 & 4 & & 2 & & & 1 & 220 \\
\hline $\begin{array}{c}\text { Pesquisa/Controle sobre } \\
\text { doenças e pragas }\end{array}$ & 78 & 137 & 254 & 60 & 18 & 11 & 4 & 1 & & & 1 & 564 \\
\hline $\begin{array}{l}\text { Eng. Genética ou } \\
\text { Melhoramento }\end{array}$ & 124 & 251 & 483 & 115 & 53 & 22 & 8 & 5 & 1 & 1 & & 1.063 \\
\hline Total & 241 & 479 & 894 & 229 & 89 & 39 & 15 & 9 & 1 & 1 & 2 & 1.999 \\
\hline
\end{tabular}

Fonte: Elaborada pelos autores deste artigo com base no Derwent Innovation Index

\subsection{Taxa de Convergência Intrasseção e Interseção}

A taxa de convergência intrasseção e interseção foi calculada por ano e comparada com o número total de documentos de patente segundo o ano de publicação (Figura 4). A taxa de convergência intrasseção apresentou uma variação entre 2,3\% e 8,36\%, mostrando seu auge em 2014, enquanto a taxa da convergência interseção variou de 1,9\% a 5,81\%, porém com um declínio a partir de 2012. Os anos de 2015 e 2016 mostram dados parciais, devido às características da base consultada e à fase de sigilo. Estes resultados sugerem que as técnicas de produção de mudas são concentradas em poucos domínios tecnológicos. 
Figura 4 - Taxas de convergências tecnológicas intrasseção e interseção dos documentos de patente sobre técnicas de produção de mudas de cana-de-açúcar segundo o ano da publicação. $\mathrm{N}=1999$. Período: 2000-2016

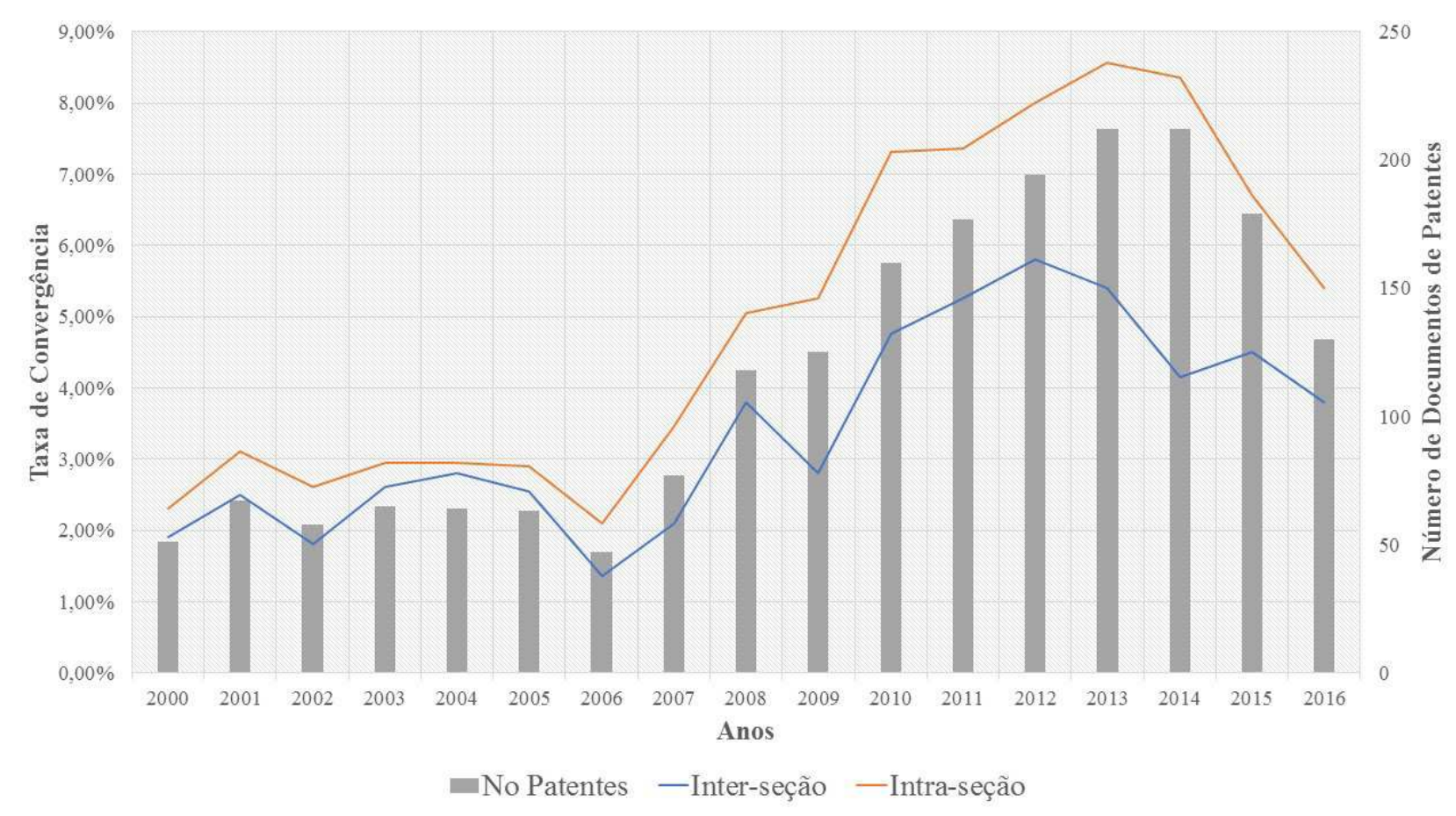

Fonte: Elaborada pelos autores deste artigo com base no Derwent Innovation Index

Para verificar o número de domínios tecnológicos nas convergências tecnológicas, os documentos de patente convergentes foram separados pelo número de domínios tecnológicos coclassificados nos níveis intrasseção (Figura 5) e interseção (Figura 6) e analisadas as interações das indexações por grupo de tecnologia (Tabela 4). O Gráfico da Figura 5 mostra a evolução da convergência tecnológica intrassseção, onde a maioria dos documentos de patente é coclassificada em dois domínios tecnológicos, os quais variam entre 45,5\% a 87,5\% de frequência frente aos demais domínios tecnológicos. Os domínios de biotecnologia e a indústria da fermentação (C06+D16) foram as classificações Derwent mais frequentes, vinculados majoritariamente à engenharia genética e melhoramento vegetal, seguidos dos domínios de compostos químicos (C03+C02) voltados para controle de doenças e pragas da cana-de-açúcar (Tabela 4). A convergência tecnológica intrasseção, composta com três domínios tecnológicos, também possui um destaque expressivo, com uma média de $15 \%$ de frequência, sendo atribuídas às classificações Derwent (C06+D16+D13) e associadas às técnicas de Engenharia Genética ou Melhoramento Vegetal (Tabela 4).

A evolução da taxa de convergência tecnológica interseção mostra uma tendência de maior crescimento após 2007, sendo composta em sua grande maioria pela indexação de classificações de duas seções diferentes (Figura 6), variando de 85,3\% a 100\% de frequência. As classificações 
Derwent mais representativas foram (C06+D16+P13), mostrando que o foco estava na produção de mudas por cultivo vegetal, utilizando técnicas de engenharia genética ou melhoramento vegetal (Tabela 4). As convergências tecnológicas interseção vinculadas com tecnologias de engenharia (T01 e T02), tais como computação digital, não tiveram uma frequência alta, o que denota que as produções de mudas por cultivo de tecidos não utilizam estas ferramentas em seu processo produtivo. Estes resultados apontam para uma grande oportunidade de inserir a produção de mudas de cana-de-açúcar na era da indústria 4.0, onde a aplicação da tecnologia digital no agronegócio poderá contribuir no ganho de produtividade.

Choi, Jeon e Kim (2005) relatam taxas de crescimento baixas de convergência tecnológica interseção nas áreas de Biotecnologia, Computação Digital e Engenharia Química, o que pode explicar o comportamento dos resultados aqui apresentados. Não obstante, observa-se que as tecnologias convergentes estão em áreas de ponta, que precisam da complementaridade disciplinar no processo de inovação. Este é o caso da biotecnologia, que mudou o paradigma tecnológico do setor agrícola, com ganhos de produtividade, redução de custos e inovações no melhoramento vegetal (BORGES; POZ, 2001). Esta tendência se afirma através dos resultados obtidos neste estudo, onde a engenharia genética e melhoramento vegetal é o principal grupo na análise de convergência tecnológica.

Figura 5 - Frequência em porcentagem do número de domínios tecnológicos da convergência intrasseção por documento de patentes sobre técnicas de produção de mudas de cana-de-açúcar ao longo do tempo. $\mathrm{N}=1999$. Período: $2000-2016$

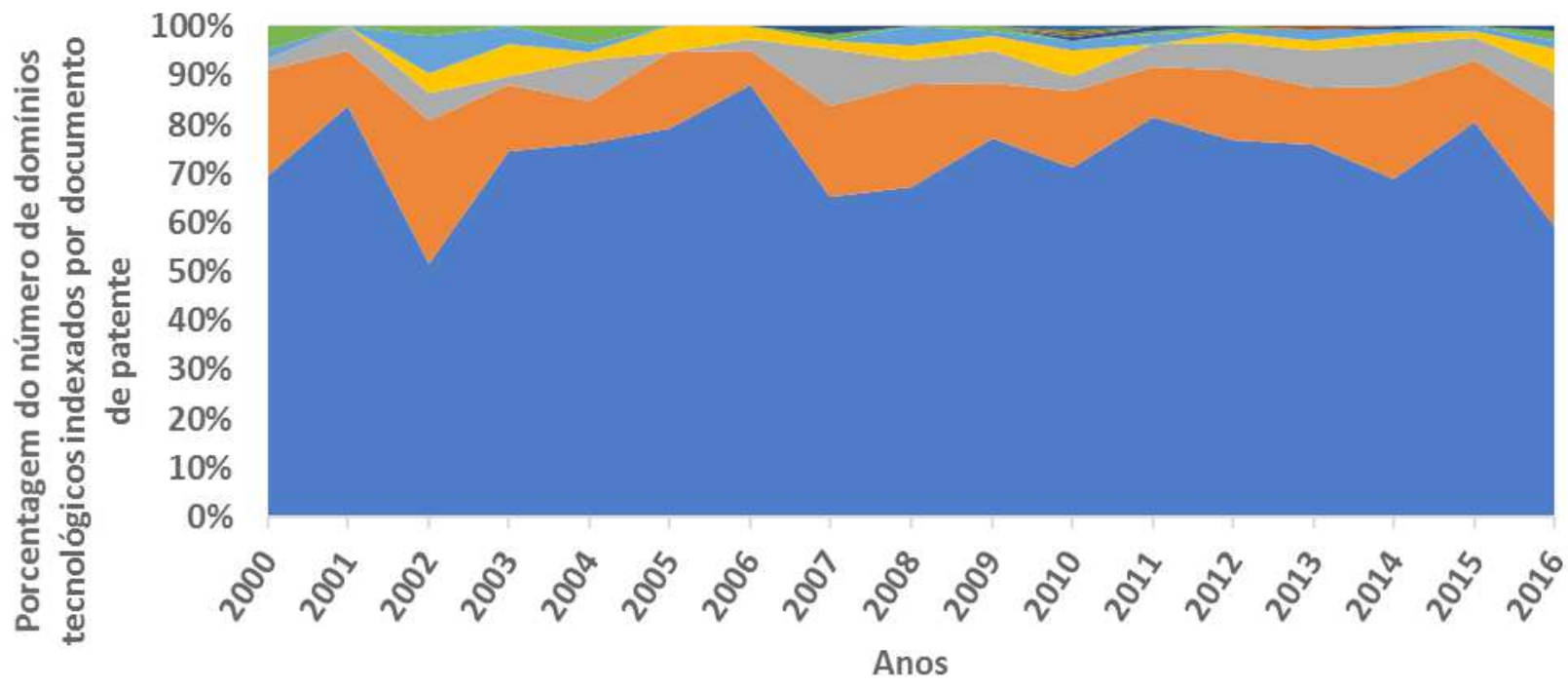

\begin{tabular}{l|l|l|l|l|l|l|l|l|l|l}
2 & 3 & 4 & 5 & 6 & 7 & 8 & 9 & 10 & 11 & 12
\end{tabular}

Fonte: Elaborada pelos autores deste artigo com base no Derwent Innovation Index 
Figura 6 - Frequência em porcentagem do número de domínios tecnológicos da convergência interseção por documento de patentes sobre técnicas de produção de mudas de cana-de-açúcar ao longo do tempo. N= 1999. Período: 2000-2016

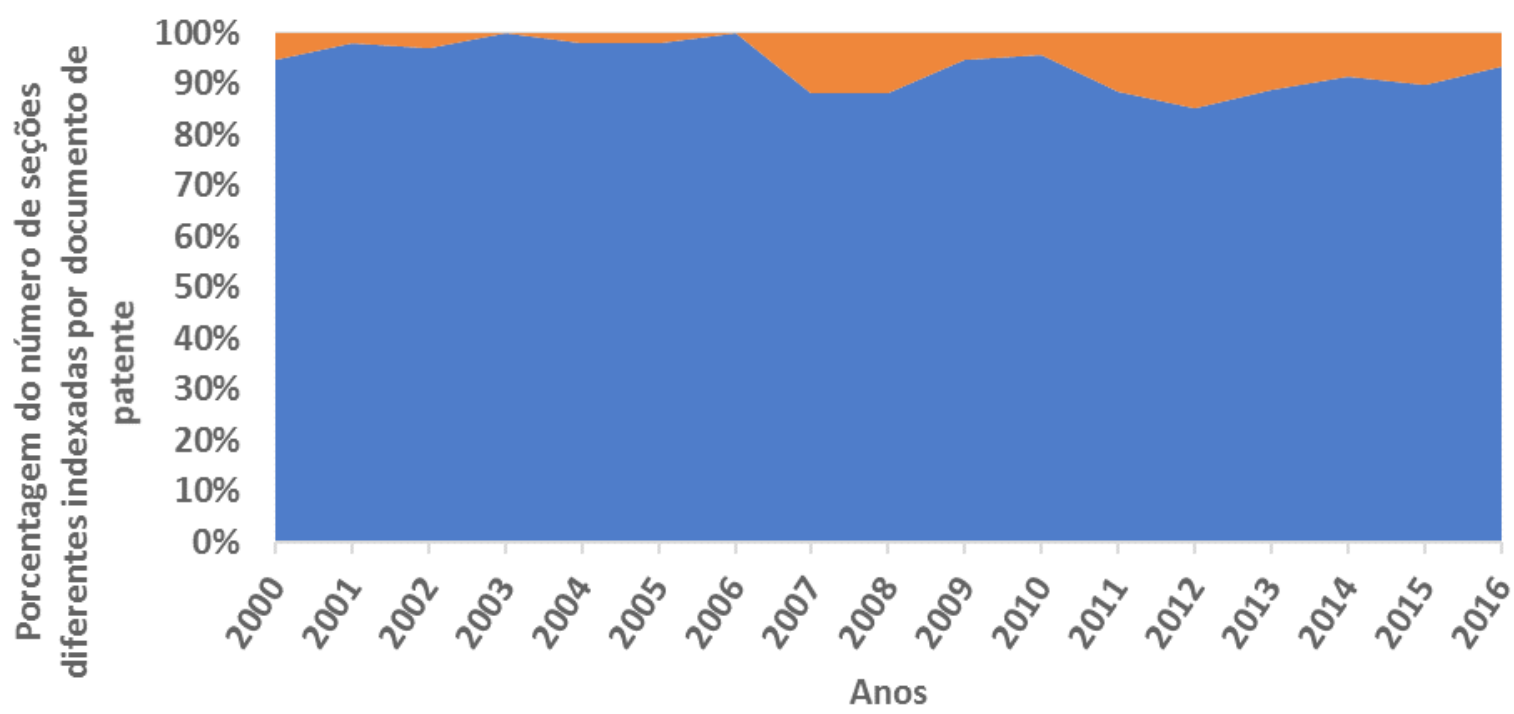

$\square 2 \square 3$

Fonte: Elaborada pelos autores deste artigo com base no Derwent Innovation Index

Tabela 4 - Principais domínios tecnológicos associados nas convergências tecnológicas intra e interseção das classificações C06 e D16 dos documentos de patente sobre técnicas de produção de mudas de cana-de-açúcar. $\mathrm{N}=1134$, Período - 2000-2016. Legenda disponível na Tabela 1

\begin{tabular}{|c|c|c|c|c|c|c|c|c|}
\hline \multirow[b]{2}{*}{$\begin{array}{l}\text { TÉCNICAS DE PRODUÇÃo DE MUDAS DE } \\
\text { CANA-DE-AÇÚCAR }\end{array}$} & \multicolumn{3}{|c|}{$\begin{array}{l}\text { INTRASSEÇÃo } \\
\text { C06+D16 }\end{array}$} & \multicolumn{4}{|c|}{ INTERSEÇÃo C06+D16 } & \multirow[b]{2}{*}{ Total } \\
\hline & VAZIO & D13 & $\mathrm{CO3}$ & P13 & P11 & T01 & T04 & \\
\hline Eng. Genética ou Melhoramento & 199 & 49 & 3 & 595 & 3 & 3 & 1 & 878 \\
\hline $\begin{array}{l}\text { Pesquisa/Controle sobre } \\
\text { doenças e pragas }\end{array}$ & 24 & 8 & 4 & 100 & 3 & 2 & & 152 \\
\hline Métodos/Pesquisa Propagação vegetativa & 9 & & 1 & 67 & & & 3 & 84 \\
\hline Demais técnicas de produção de mudas & 3 & 0 & 0 & 16 & 0 & 0 & 0 & 20 \\
\hline Total Geral & 235 & 57 & 8 & 778 & 6 & 5 & 4 & 1.134 \\
\hline
\end{tabular}

Fonte: Elaborada pelos autores deste artigo com base no Derwent Innovation Index

\section{Considerações Finais}

A metodologia de análise de coclassificação de patentes utilizando a classificação do Derwent mostrou ser simples para estudos de convergências tecnológicas. Apesar das baixas taxas de convergência tecnológica relacionadas com as técnicas de produção de mudas de cana-de-açúcar, a pesquisa mostrou a relação entre diferentes domínios tecnológicos. A multidisciplinaridade 
está vinculada à indústria da fermentação, biotecnologia e cultivo vegetal, mostrando que o direcionamento da indústria canavieira está voltado para o melhoramento vegetal e produção de mudas com alta tecnologia. No entanto, vislumbra-se uma grande oportunidade de aliar a produção de mudas de cana com tecnologias digitais da indústria 4.0, o que poderá proporcionar um diferencial competitivo no agronegócio canavieiro.

Esta metodologia pode ser facilmente aplicada a estudos de diferentes áreas tecnológicas e adaptada para qualquer tipo de classificação de patentes, o que pode ajudar na análise dos processos de inovação, suas complexidades e dificuldades na complementaridade multidisciplinar.

\section{Referências}

BORGES, I. D. C.; POZ, D. Impactos da Biotecnologia Moderna no Agronegócio Brasileiro. p. 1-20, 2001.

CHOI, J. Y.; JEONG, S.; KIM, K. A study on diffusion pattern of technology convergence: Patent analysis for Korea. Sustainability (Switzerland), v. 7, n. 9, p. 11.546-11.569, 2015.

CURRAN, C.; BRÖRING, S.; LEKER, J. Technological Forecasting \& Social Change Anticipating converging industries using publicly available data. Technological Forecasting \& Social Change, v. 77, n. 3, p. 385-395, 2010. Disponível em: <http://dx.doi.org/10.1016/j.techfore.2009.10.002>. Acesso em: 12 abr. 2017.

JANNUZZI, A. H. L.; AMORIM, R. D. C. R.; SOUZA, C. G. DE. Implicações da categorização e indexação na recuperação da informação tecnológica contida em documentos de patentes. Ciência da Informação, v. 36, n. 2, p. 27-34, 2007.

JEONG, S.; KIM, J. C.; CHOI, J. Y. Technology convergence: What developmental stage are we in? Scientometrics, v. 104, n. 3, p. 841-871, 2015.

RUAS, T. L.; PEREIRA, L. Como construir indicadores de Ciência, Tecnologia e Inovação usando Web of Science, Derwent World Patent Index, Bibexcel e Pajek? Perspectivas em Ciência da Informação, p. 52-81, 2014.

SMITH, M. et al. NodeXL:L: a free and open network overview, discovery and exploration add-in for Excel 2007/2010/2013/2016. [S.1.]: Social Media Research Foundation (<http://www.smrfoundation. org >). 2010. Disponível em: <http://nodexl.codeplex.com/> . Acesso em: 12 abr. 2017.

SNYMAN, S. J. et al. Applications of in vitro culture systems for commercial sugarcane production and improvement. In Vitro Cellular and Developmental Biology - Plant, v. 47, n. 2, p. 234-249, 2011.

SONG, C. H.; ELVERS, D.; LEKER, J. Anticipation of converging technology areas ??? A refined approach for the identification of attractive fields of innovation. Technological Forecasting and Social Change, v. 116, p. 98-115, 2017.

VAN ECK, N. J.; WALTMAN, L. VOSviewer - Visualizing scientific landscapes . [S.l: s.n.]. Disponível em: <http://www.vosviewer.com/>. Acesso em: 12 abr. 2017. 


\section{Sobre os autores}

\section{Cecilia Hasner}

E-mail: cecilia@prospective.com.br

Mestre e Doutora em propriedade intelectual e inovação (INPI). Especialista em gestão ambiental.

Endereço Profissional: Rua Vinte 230, sala 4, Santa Monica, CEP: 29.105-400, Vila Velha, ES.

\section{Eduardo Winter}

E-mail: winter@inpi.gov.br

Graduado em Química Industrial pela Pontifícia Universidade Católica do Paraná. Mestre em Química pela Universidade Estadual de Campinas. Doutor em Ciências pela Universidade Estadual de Campinas.

Endereço Profissional: Rua Mayrink Veiga, 09/17 andar, Centro - CEP: 20.090-910, Rio de Janeiro, RJ.

\section{Araken Alves de Lima}

E-mail: araken@inpi.gov.gr

Doutor em Economia na Unicamp. Atua na Coordenação Geral da Disseminação para a Inovação - CGDI, no Instituto Nacional da Propriedade Industrial - INPI de Santa Catarina.

Endereço Profissional: Rua Nunes Machado, 192, centro, Florianópolis - SC. CEP 88010-460. 